\title{
Are CSF Biomarkers Useful as Prognostic Indicators in Diagnostically Unresolved Cognitively Impaired Patients in a Normal Clinical Setting?
}

\author{
Malene Schjønning Nielsen ${ }^{a} \quad$ Anja Hviid Simonsen ${ }^{b} \quad$ Volkert Siersma $^{c}$ \\ Steen Gregers Hasselbalch ${ }^{b}$ Peter Høgh ${ }^{a}$ \\ ${ }^{a}$ Regional Dementia Research Centre, Department of Neurology, Zealand University \\ Hospital, Roskilde, and ' ${ }^{b}$ Danish Dementia Research Centre, Department of Neurology, \\ Rigshospitalet, University of Copenhagen, and 'The Research Unit for General Practice \\ and Section of General Practice, Department of Public Health, University of Copenhagen, \\ Copenhagen, Denmark
}

Key Words

Alzheimer disease · Mild cognitive impairment · Biomarker · Cerebrospinal fluid · Amyloid ·

Tau protein · Diagnosis · Prognosis · Blood-brain barrier · Inflammation

\begin{abstract}
Background: Despite an extensive evaluation program, patients may remain diagnostically unresolved with regard to the etiology of their cognitive dysfunction. Cerebrospinal fluid neuroinflammation and Alzheimer disease (AD) biomarkers may act as indicators of neurodegenerative disorders in diagnostically unresolved patients. Methods: Data on 348 patients were retrospectively evaluated. All participants had a standardized diagnostic workup and followup in a memory clinic. Results: $A \beta_{42}$ levels and $A \beta_{42} / p$-tau ratios were reduced and levels of $\mathrm{t}$-tau and $\mathrm{p}$-tau as well as the t-tau $\times \mathrm{p}$-tau/A $\beta_{42}$ ratio were elevated in diagnostically unresolved patients who clinically progressed, compared to a stable group. No differences in neuroinflammatory parameters were found. Conclusion: AD biomarkers - in particular the $A \beta_{42} /$ p-tau ratio, but not neuroinflammatory parameters - predicted clinical progression, regardless of etiology.




\section{Introduction}

Dementia is a massive and growing global health-economic challenge with an estimated prevalence of 35.6 million people affected worldwide in 2010. A continuous increase in the prevalence of dementia is to be expected [1].

It is therefore of great importance to identify patients who suffer from cognitive impairment due to a neurodegenerative disease in order to implement proper treatment and care early in the course of the disease, as these patients will continue to deteriorate. It is equally important to identify in which cases Alzheimer disease (AD) is the cause of the cognitive impairment, as this patient group is by far the largest to whom symptomatic treatment can be offered. Likewise, an identification of patients with a high probability of progression early in the course of the disease is becoming increasingly important for clinical trials of disease-modifying agents. Treatment should preferably be initiated early in the course of the disease, when neuronal damage is still limited [2].

Frequently, patients referred for cognitive evaluation may still remain diagnostically unresolved with regard to the etiology of their cognitive dysfunction even after an extensive diagnostic workup including cerebrospinal fluid (CSF) biomarker analysis. These diagnostically unresolved patients may be diagnosed with either mild cognitive impairment (MCI) or as demented with unknown etiology. The critical issue is to differentiate the underlying etiology in order to predict progression of the condition.

Not every case of MCI will progress to a dementia disorder; however, $10-15 \%$ of patients with MCI will develop AD within 1 year, and even 40-60\% will develop AD within 5 years [3-5]. The concept of 'dementia of unknown etiology' (DUE) has been used to describe demented patients who do not meet the criteria for any well-defined degenerative dementia such as $\mathrm{AD}$, Lewy body dementia, frontotemporal dementia, or other causes of secondary dementia (e.g. vitamin $\mathrm{B}_{12}$ deficiency or head trauma) [6].

The diagnosis of dementia is currently based on different sets of clinical diagnostic criteria, of which the NIA-AA criteria and ICD-10 criteria are generally accepted [7, 8]. Various biomarkers, in particular CSF biomarkers, have proven useful in increasing the diagnostic certainty in vivo, and findings from biomarker analyses have been incorporated into different research criteria for $\mathrm{AD}$ [9] but are also gaining increasing attention in clinical practice.

Potential biomarkers of cognitive decline and dementia need to exhibit a high diagnostic accuracy and should reflect the biochemical and pathological hallmarks of the disease. At present, known biomarkers still lack sufficient diagnostic accuracy [10].

$\mathrm{CSF}$ analysis may to a large extent support the diagnosis if AD is the underlying pathology, but $\mathrm{AD}$ biomarkers (i.e. $\mathrm{A} \beta_{42}$, t-tau, and p-tau) cannot definitely confirm the diagnosis even in the presence of typical findings, since these alterations may also be present in cognitively healthy persons as well as in patients with other neurodegenerative disorders [10-15]. However, decreased levels of CSF $A \beta_{42}$ and increased levels of t-tau and p-tau confer a high likelihood of $\mathrm{AD}$ being the cause of the cognitive dysfunction in both dementia and MCI [16].

There is an increasing interest in the involvement of neuroinflammation in the development and progression of dementia. Evidence of this involvement derives from pathophysiologic studies, which have revealed a number of inflammatory mediators to be upregulated in the $\mathrm{AD}$ brain $[10,17,18]$. Other studies have suggested that the presence of amyloid plaques and neurofibrillary tangles has the ability to initiate and sustain a chronic inflammatory response via the complement system during the disease $[19,20]$. The CSF/serum albumin ratio, as an indicator of blood-brain barrier (BBB) integrity, has a limited differential diagnostic power, as it may be affected in both neuroinflammatory diseases as well as in cerebrovascular lesions; however, it is generally found to be a reliable sign of an active process [2124]. Elevated CSF/serum albumin ratio, white cell count, and intrathecal IgG synthesis may 
therefore be potential markers of neuroinflammatory processes, which implies that analysis of theses markers in CSF can help to improve diagnostic accuracy and predict prognosis in patients referred for dementia evaluation.

In our study, we aimed to investigate the role of CSF neuroinflammation and AD biomarkers in the diagnostic workup for a group of diagnostically unresolved patients in a typical memory clinical setting. In this group, despite an extensive evaluation program including CSF biomarker analysis, experienced clinicians were not able to establish whether a neurodegenerative disease was the cause of the cognitive impairment or not. Hence, we investigated the biomarkers' ability to predict clinical progression, and thereby their ability to potentially differentiate between neurodegenerative and nonneurodegenerative disorders as causes of cognitive impairment in diagnostically unresolved patients. Furthermore, we compared the diagnostically unresolved patients to a control group of $\mathrm{AD}$ patients as well as a group of healthy elders to elucidate similarities.

\section{Subjects and Methods}

Data were retrospectively collected from the Danish Dementia Biobank on patients consecutively referred for cognitive evaluation and diagnosed with either MCI, DUE, or AD at 2 multidisciplinary memory clinics (Regional Dementia Research Centre, Department of Neurology, Zealand University Hospital, and Copenhagen Memory Clinic, Department of Neurology, University Hospital Copenhagen, Rigshospitalet) between June 2009 and June 2013. The study population also included a healthy control (HC) group recruited solely for research purposes.

As part of the diagnostic workup, at baseline all patients underwent a standardized diagnostic assessment including physical and neurological examinations, routine blood analysis, lumbar puncture with CSF analysis, brain CT or MRI, as well as cognitive screening [i.e. MiniMental State Examination (MMSE) and Addenbrooke's Cognitive Examination (ACE)]. If it was thought diagnostically relevant, the patients also had a full neuropsychological evaluation undertaken by a neuropsychologist. Diagnoses were settled by consensus in an experienced multidisciplinary team based on all the available examination results, including the results from the CSF analysis. Patients with AD were diagnosed according to the NINCDSADRDA criteria (2009-2011), subsequently according to the NIA-AA criteria [7, 9]. Patients with MCI were diagnosed according to the Winblad consensus criteria [25]. Patients with DUE were those diagnosed as being demented according to the ICD-10 and NIA-AA criteria $[7,8]$ but who did not fulfill the diagnostic criteria for any subtype of dementia or secondary dementia because the underlying etiology could not be identified.

All patients were clinically evaluated - including repeated MMSE testing and information from caregivers - on an ongoing basis with at least 1-year intervals as part of the normal clinical setting in the 2 multidisciplinary memory clinics. The diagnoses were continuously reevaluated at the follow-up visits. Based on clinical progression, to fulfill the criteria for AD or other well-defined dementia disease, the diagnoses were subsequently revised by an experienced physician or at a multidisciplinary conference.

The healthy subjects were all volunteers recruited for scientific research purposes. They were all without memory complaints or other cognitive symptoms as well as signs of major neurological, psychiatric, or physical diseases that could potentially elicit cognitive impairment. The healthy subjects all underwent the same standardized diagnostic assessment in one of the 2 memory clinics, including physical and neurologic examinations, routine blood analysis, brain CT or MRI, cognitive screening, as well as blood sample screening, ECG, and lumbar puncture at inclusion. 
CSF was obtained by puncture in the L3-L4 or L4-L5 intervertebral space and collected in polypropylene tubes. The CSF analyses included white cell count, erythrocytes, total protein, glucose, and albumin as well as IgG index and oligoclonal bands and the core AD biomarkers (i.e. $\mathrm{A} \beta_{42}, \mathrm{t}$-tau, and $\mathrm{p}$-tau). The $\mathrm{CSF} /$ serum albumin ratio was used as a marker of the integrity of the BBB, due to the fact that albumin is exclusively synthesized in the liver [21-24]. The CSF/serum albumin ratio is therefore a dimensionless CSF protein concentration, independent of blood variations [22]. The IgG index was used to determine IgG synthesis, and along with the CSF white cell count was used as an indicator of active neuroinflammation as well as a marker of BBB integrity.

For the purposes of this study, patients initially diagnosed with MCI or DUE were combined into a group of patients defined as diagnostically unresolved. Based on the ongoing clinical evaluation, the diagnostically unresolved patients were subsequently divided into a group of patients who progressed during follow-up to eventually fulfill the criteria for a welldefined dementia disease and a group of stable patients who did not progress or who were diagnosed with a nonneurodegenerative disorder.

The study was reported and approved by the Data Protection Agency authorities. As the study is a retrospective study exclusively analyzing data from the Danish Dementia Biobank, no specific ethics committee approval was needed according to local legislation.

Comparisons between groups were performed using the nonparametric KruskalWallis test followed by the Mann-Whitney U test for pairwise comparisons. To correct for multiple comparisons, results were considered statistically significant if $\mathrm{p}$ values were $<0.01$. Receiver operating characteristic (ROC) curves of each CSF parameter and biomarker individually as well as in combination were drawn as a plot of the true-positive value (sensitivity) against the false-positive value (1 - specificity) for different possible cutoff values of the respective markers. The ROC curves illustrate the ability of each biomarker or combination of biomarkers to correctly classify progressive and stable patients. The area under the curve (AUC) was calculated as a measure of a biomarker's overall accuracy. Optimized cutoff levels for AD biomarkers individually and in combination were derived from the ROC curves by the cutoff that maximized the sum of sensitivity and specificity. Previous studies have found a clear correlation between age and t-tau level, which makes it necessary to determine separate cutoff values for different age categories [26]. In our study, only 4 patients were under the age of 45 years at baseline; hence, age stratification was not performed. We also used previously reported reference values for CSF A $\beta_{42}$, t-tau, and p-tau to evaluate how they performed in predicting clinical progression in a population of diagnostically unresolved patients (table 3) [27, 28]. The statistical analysis was carried out using SPSS version 22 and the R environment for statistical computing version 3.1.2.

\section{Results}

A total of 348 patients were included. They were either diagnosed with MCI $(n=60)$, DUE ( $\mathrm{n}=83)$, or AD $(\mathrm{n}=172)$ or as HC $(\mathrm{n}=33)$. The group of diagnostically unresolved patients (the MCI and DUE groups) included 143 patients.

Seventy-two of the 143 diagnostically unresolved patients remained clinically stable during the follow-up period (mean follow-up time 21.4 months, range 1-59), and 71 patients progressed to fulfill the criteria for a well-defined dementia disease. Seven patients in the stable group had a follow-up period of less than 1 year due to the identification of nonneurodegenerative conditions during clinical follow-up (e.g. depression, cerebral infarction) or full recovery. 
Table 1. Demographic data and cognitive scores at baseline

\begin{tabular}{|c|c|c|c|c|}
\hline & $\begin{array}{l}\text { Progressive, } \\
\text { diagnostically } \\
\text { unresolved }\end{array}$ & $\begin{array}{l}\text { Stable, } \\
\text { diagnostically } \\
\text { unresolved }\end{array}$ & $\mathrm{AD}$ & $\mathrm{HC}$ \\
\hline Subjects, n (\%) & $71(20.4)$ & $72(20.7)$ & $172(49.4)$ & $33(9.5)$ \\
\hline MCI/DUE, \% & $42 / 58$ & $35 / 65$ & & \\
\hline Age, years & $68.2 \pm 8.5(50-85)$ & $67.7 \pm 9.2(35-85)$ & $68.1 \pm 9.4(36-90)$ & $66.6 \pm 7.5(51-85)$ \\
\hline \multicolumn{5}{|l|}{ Gender, \% } \\
\hline Male & 49.3 & 62.5 & 55.2 & 54.5 \\
\hline Female & 50.7 & 37.5 & 44.8 & 45.5 \\
\hline MMSE score & $24.7 \pm 4.0^{\mathrm{a}, \mathrm{b}}$ & $25.7 \pm 3.9^{a}$ & $23.0 \pm 4.5^{\mathrm{a}}$ & $29.2 \pm 1.0$ \\
\hline Follow-up, months & $23.0 \pm 13.9(1-61)$ & $21.4 \pm 14.6(1-59)$ & $18.5 \pm 11.8(1-59)$ & $24.3 \pm 3.4(14-37)$ \\
\hline
\end{tabular}

Values are given as mean \pm standard deviation (range), unless specified otherwise. Results are considered significant when $\mathrm{p}<0.01$, due to multiple comparisons. MMSE = Mini-Mental State Examination; $\mathrm{MCI}=$ mild cognitive impairment; DUE =

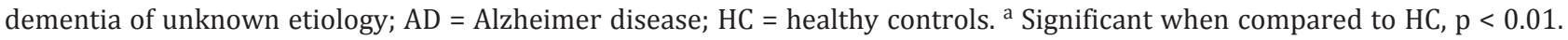
b Significant when compared to AD, $\mathrm{p}<0.01$.

Table 2. CSF inflammation and AD biomarker levels and ratios at baseline

\begin{tabular}{|c|c|c|c|c|}
\hline & $\begin{array}{l}\text { Progressive, } \\
\text { diagnostically unresolved }\end{array}$ & $\begin{array}{l}\text { Stable, } \\
\text { diagnostically unresolved }\end{array}$ & $\mathrm{AD}$ & $\mathrm{HC}$ \\
\hline $\mathrm{A} \beta_{42}, \mathrm{pg} / \mathrm{ml}$ & $343(245-460)^{\mathrm{a}, \mathrm{c}}$ & $404(269-733)^{\mathrm{b}, \mathrm{c}}$ & $302(191-446)^{c}$ & $925(849.5-1,249)$ \\
\hline t-tau, pg/ml & $53(39-79)^{\mathrm{a}}$ & $43(26.5-54.5)^{\mathrm{b}}$ & $64(43-92)$ & $45(30-61.5)$ \\
\hline $\mathrm{p}$-tau, pg/ml & $340(230-570)^{\mathrm{a}}$ & $223(143.5-298.5)^{b}$ & $378(213-610)^{\mathrm{c}}$ & $281(202.5-359.5)$ \\
\hline $\mathrm{A} \beta_{42} / \mathrm{p}$-tau & $7.0(3.6-11.1)^{\mathrm{a}-\mathrm{c}}$ & $11.5(8.2-18.3)^{\mathrm{b}, \mathrm{c}}$ & $4.4(2.4-8.9)^{\mathrm{c}}$ & $23.8(17.6-29.4)$ \\
\hline t-tau $\times p-\operatorname{tau} / A \beta_{42}$ & $49.4(26.0-143.4)^{\mathrm{a}, \mathrm{c}}$ & $17.4(9.2-36.6)^{b}$ & $81.4(26.0-210.6)^{c}$ & $11.7(8.7-22.7)$ \\
\hline White cell count & $1.0(0.0-2.0)^{\mathrm{b}}$ & $3.0(2.0-4.0)$ & $3.0(2.0-5.0)$ & $3.0(1.0-4.0)$ \\
\hline IgG index & $0.49(0.45-0.57)$ & $0.48(0.46-0.57)$ & $0.52(0.46-0.57)$ & $0.51(0.48-0.57)$ \\
\hline $\mathrm{CSF} /$ serum albumin & $0.007(0.005-0.008)$ & $0.006(0.005-0.009)$ & $0.006(0.004-0.008)$ & $0.006(0.004-0.009)$ \\
\hline
\end{tabular}

Values are given as median (interquartile range). Results are considered significant when $\mathrm{p}<0.01$, due to multiple comparisons. CSF $=$ Cerebrospinal fluid;

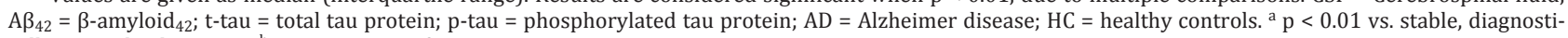
cally unresolved patients. ${ }^{\mathrm{b}} \mathrm{p}<0.01$ vs. AD. ${ }^{\mathrm{c}} \mathrm{p}<0.01$ vs. HC.

The descriptive data on diagnosis, age, gender, baseline MMSE scores, and follow-up period are presented in table 1. No significant group differences in mean age were found. As expected, the baseline MMSE scores were significantly higher in the HC group than in all the other groups $(\mathrm{p}<0.01)$. The AD group had significantly lower baseline MMSE scores than the clinically progressive group $(p<0.01)$, but not than the clinically stable group $(p=0.016)$. No significant differences in MMSE score were observed between the progressive and the stable group.

Table 2 illustrates the baseline CSF inflammation and AD biomarker levels and ratios among the diagnostic groups. The concentration of CSF $\mathrm{A} \beta_{42}$ was significantly lower in patients who clinically progressed during the follow-up period than in the clinically stable patients and the $\mathrm{HC}$, but it was not significantly different from that of the AD group. The clinically stable group had a significantly lower baseline CSF $A \beta_{42}$ level than the $\mathrm{HC}$, but a higher level than the AD group. As expected, the HC group had a significantly higher baseline CSF $A \beta_{42}$ level than all the other groups. With regard to tau protein, the clinically progressive group had significantly higher levels of both CSF t-tau and p-tau protein than the stable group, but it did not significantly differ in either t-tau or p-tau levels from the AD or the HC group. The stable group had significantly lower levels of CSF t-tau and p-tau protein than the AD 


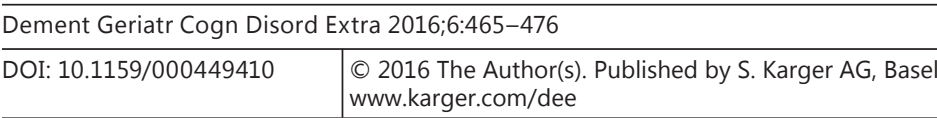

Schjønning Nielsen et al.: Are CSF Biomarkers Useful as Prognostic Indicators in Diagnostically Unresolved Cognitively Impaired Patients in a Normal Clinical Setting?

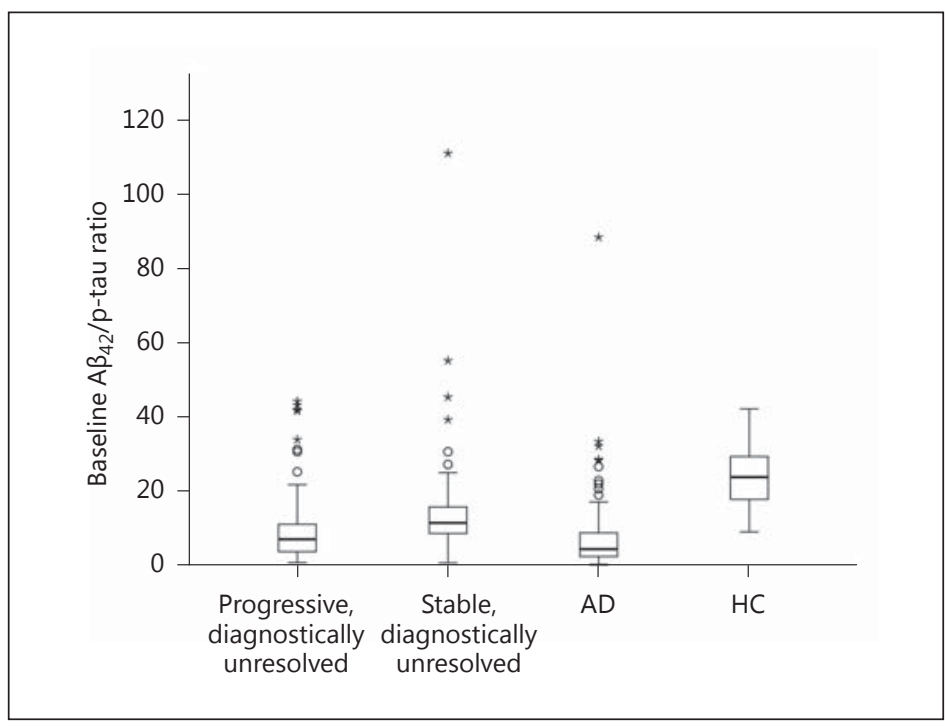

Fig. 1. Baseline CSF $A \beta_{42} / \mathrm{p}$-tau ratio categorized into: progressive, diagnostically unresolved; stable, diagnostically unresolved; $\mathrm{AD}$; and HC. The line in the box represents the median; the lower and upper box bounds mark the 1st and 3rd quartiles. The 95\% confidence interval is marked by whiskers, and open circles and stars represent outliers. The $\mathrm{A} \beta_{42} / \mathrm{p}$-tau ratio differed significantly between all the groups $(\mathrm{p}<0.01)$.

Fig. 2. Baseline CSF t-tau $\times \mathrm{p}$-tau/ $\mathrm{A} \beta_{42}$ ratio categorized into: progressive, diagnostically unresolved; stable, diagnostically unresolved; $\mathrm{AD}$; and $\mathrm{HC}$. The line in the box represents the median; the lower and upper box bounds mark the 1st and 3rd quartiles. The 95\% confidence interval is marked by whiskers, and open circles and stars represent outliers. The t-tau $\times \mathrm{p}$-tau $/ \mathrm{A} \beta_{42}$ ratio differed significantly when the clinically progressive group was compared to the clinically stable group and the HC $(\mathrm{p}<0.01)$. A significant difference was also found between the $\mathrm{AD}$ group and the clinically stable patients subsequent to the HC $(\mathrm{p}<0.01)$.

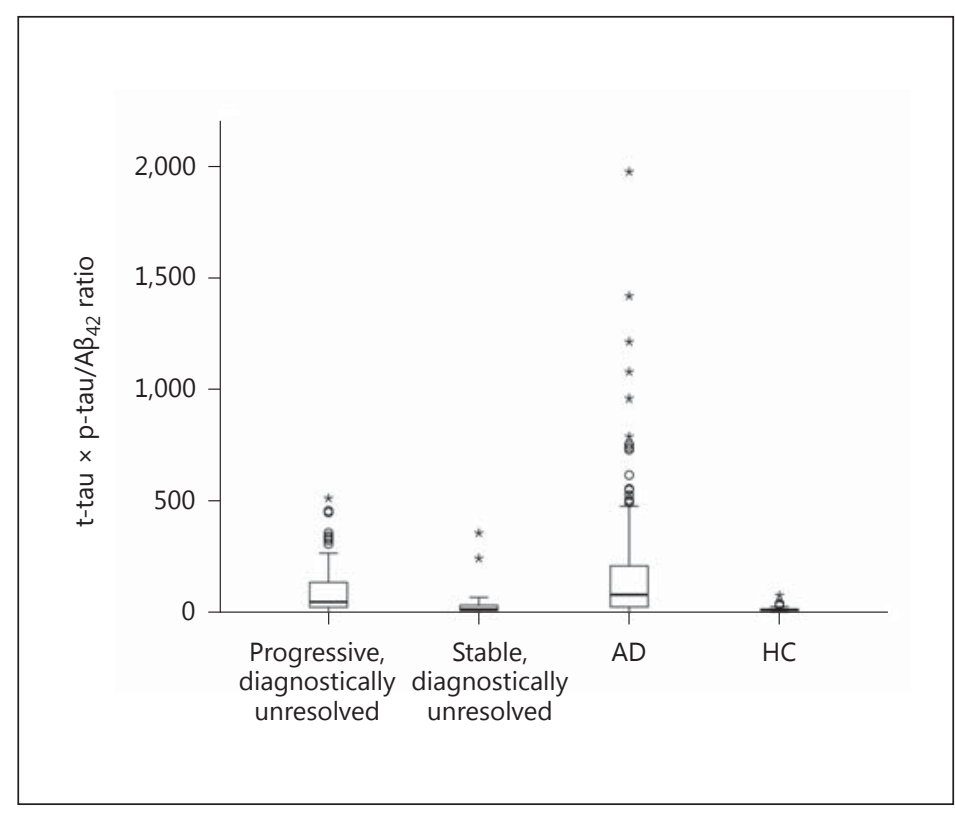

group, but no significant differences in either level were found compared to the HC group. The HC group had only a significantly lower CSF p-tau level, but not CSF t-tau level, than the AD group. The CSF inflammation biomarkers (i.e. white cell count, IgG index, and CSF/serum albumin ratio) did not show any significant differences when comparing the clinically progressive, the clinically stable, and the HC group. Only a significantly higher CSF white cell count was found in the AD group when compared to the clinically progressive group. This can be attributed to a few outlier patients with highly elevated CSF white cell counts only in the AD group. Our data showed great variability in the few patients with an elevated white cell count and may therefore not be representative of the different patient groups.

The CSF A $\beta_{42} / p$-tau ratio differed significantly between all groups when comparing the groups pairwise. The ratio was significantly lower in both patients who progressed clinically 
Fig. 3. ROC curves and corresponding AUCs for the ability of CSF AD and neuroinflammation biomarkers to predict future progression in our study population. The $A \beta_{42} / p$-tau and t-tau $\times$ p-tau/ $\mathrm{A} \beta_{42}$ ratios performed better than the CSF biomarkers individually in predicting future progression.

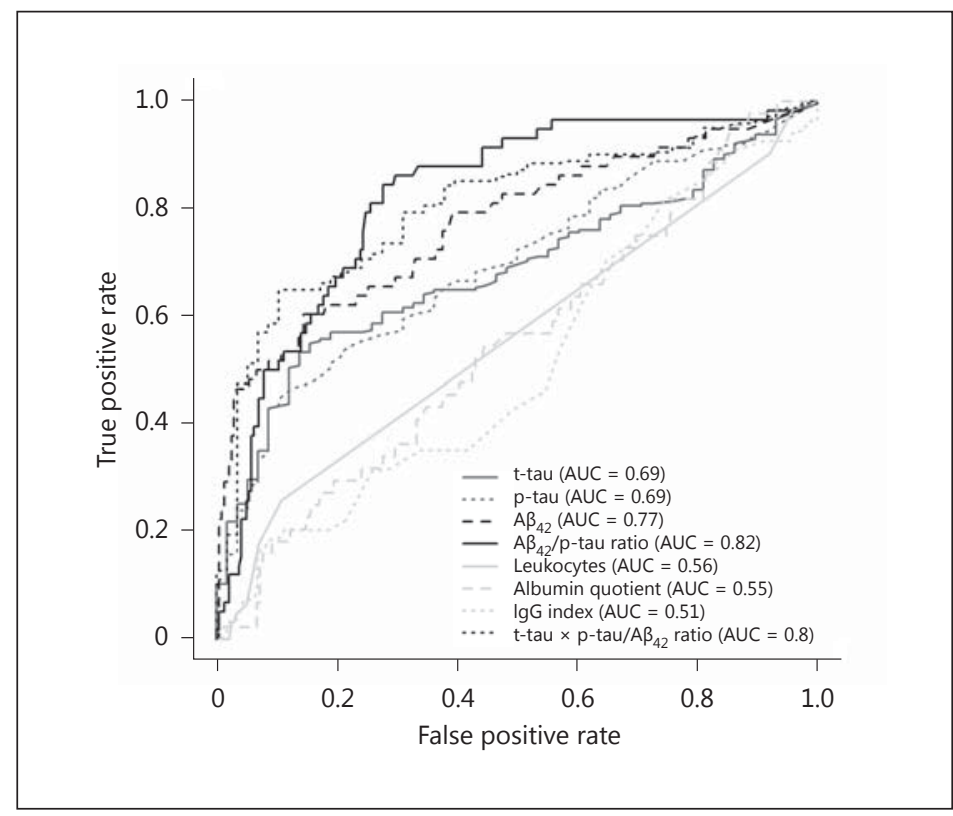

and patients with AD when compared to clinically stable patients and HC. The CSF A $\beta_{42} / p$-tau ratio was moreover significantly higher in the progressive group than in the AD group. Similarly, a higher CSF $\mathrm{A} \beta_{42} / \mathrm{p}$-tau ratio was seen in the HC than in the clinically stable patients, as illustrated in figure 1 and table 2 .

We calculated the CSF $t$-tau $\times \mathrm{p}$-tau $/ \mathrm{A} \beta_{42}$ ratio for each diagnostic group. The ratio was significantly higher in the clinically progressive group than in both the clinically stable group and the HC. No significant difference was found between the clinically progressive and the AD group. Similarly, a higher ratio was found in the AD group than in the clinically stable patients and HC. No significant difference was observed between the clinically stable group and the HC, as illustrated in figure 2 and table 2.

ROC curves are shown in figure 3. Individual AUCs for the different CSF AD biomarkers as predictors of future progression ranged from 0.69 for $t$-tau and $p$-tau to 0.77 for $A \beta_{42}$, indicating that CSF $A \beta_{42}$ had the highest predictive value of the $3 \mathrm{AD}$ biomarkers. A better performance in predicting future progression in our group of diagnostically unresolved patients was found for CSF AD biomarkers in combination: $A \beta_{42} / p$-tau ratio $(A U C=0.82)$ and $t$-tau $\times$ $\mathrm{p}$-tau $/ \mathrm{A} \beta_{42}$ ratio $(\mathrm{AUC}=0.80$ ). The ROC analysis revealed a low prognostic accuracy for CSF inflammation biomarkers (i.e. $\mathrm{CSF}$ white cell count: $\mathrm{AUC}=0.56$; $\mathrm{CSF} /$ serum albumin ratio: $\mathrm{AUC}=0.56$; $\mathrm{CSF}$ IgG index: $\mathrm{AUC}=0.51$ ).

\section{Discussion}

The main objective of our study was to investigate the role of CSF inflammation and AD biomarkers in the diagnostic workup in a group of diagnostically unresolved patients in a routine clinical setting. This is highly relevant to clinicians in a normal clinical setting, as they frequently meet these unresolved patients.

In particular, we aimed to investigate how CSF inflammation and AD biomarkers performed as well as to study their ability to predict clinical progression - and thereby their ability to identify neurodegenerative versus nonneurodegenerative disorders as causes of cognitive impairment in diagnostically unresolved patients. 
In our study, we found that those diagnostically unresolved patients who clinically progressed to fulfill the criteria for a well-defined dementia due to a neurodegenerative disease had a biomarker profile characteristic of $\mathrm{AD}$ at baseline which reflected the neuropathological hallmarks of $\mathrm{AD}$ with progressive accumulation of $\mathrm{A} \beta$ plaques, neurofibrillary tangles, and neuronal degeneration [13, 29-31]. In contrast, a similar biomarker profile was not seen in the stable diagnostically unresolved patients.

Therefore, it is very possible that the majority of the progressive diagnostically unresolved patients in our group in fact represent patients with underlying AD, but with initially atypical or subtle signs and symptoms. Some may, however, also suffer from another neurodegenerative dementia, since the CSF biomarkers' performance in distinguishing between different forms of dementia is less solid [31-34].

Previous studies have demonstrated that $\mathrm{AD}$ biomarkers have the ability to differentiate between $\mathrm{AD}$ patients and healthy elders with high sensitivity and specificity (80-90\%); also, an abnormal biomarker pattern in MCI may predict progression to AD in $90 \%$ of MCI cases within 9.2 years $[32,33,35]$. The AD biomarkers assessed in our group of diagnostically unresolved patients exhibited a slightly lower ability to accurately predict future progression, with diagnostic accuracies ranging from 0.69 to 0.7 (fig. 3). This may partly be explained by the fact that we exclusively analyzed whether cognitively impaired patients progressed to neurodegenerative dementia or not, which included all degenerative disorders and not only AD.

The CSF $A \beta_{42}$ level had the highest predictive ability of the 3 well-established AD biomarkers, which supports the theory of amyloid positivity being a risk factor for developing $\mathrm{AD}$ as well as the notion that AD biomarkers become abnormal in a temporally ordered manner in which t-tau and p-tau protein are usually not abnormal until later in the course of $\mathrm{AD}[12,32,36-38]$.

The notion of combining 2 aspects of $A D$ pathology - i.e. plaques $\left(A \beta_{42}\right)$ and neurodegeneration ( $\mathrm{p}$-tau) - as a biomarker of continuous cognitive decline and clinical progression to $\mathrm{AD}$ in MCI patients has shown promising results [32, 35, 39]. In particular, the CSF $A \beta_{42} / p$-tau ratio exhibited a great capacity to predict progression in MCI patients younger than 70 years, probably due to the lower importance of comorbidities when compared to older groups [35]. However, to our knowledge, similar findings have not previously been described for a mixed group of diagnostically unresolved patients.

Our findings with regard to the $\mathrm{CSF} A \beta_{42} / \mathrm{p}$-tau ratio suggest that it may also be a useful predictor of future clinical progression in a heterogeneous group of diagnostically unresolved patients in a normal clinical setting. ROC analysis did indeed reveal that the ratio outperformed the prevalent biomarkers individually (fig. 3). It is noteworthy though that the mean age in all groups of our study was in fact below 70 years, which could partly explain the considerable potential of the $A \beta_{42} / p$-tau ratio as a prognostic indicator $[32,34,35,39-41]$.

Furthermore, of all the biomarkers, only the $A \beta_{42} / p$-tau ratio had a significant discriminative power to separate all the groups individually. However, supposing the $A \beta_{42} / p$-tau ratio were to be incorporated as a useful diagnostic tool into a normal clinical setting with a heterogeneous group of cognitively impaired patients, generally accepted cutoff values for demented populations would need to be established [33].

The optimized cutoff values derived from the ROC curves for the individual AD biomarkers were similar to well-established reference values (table 3) [27, 28, 42]. Our optimized cutoff value for the $A \beta_{42} / p$-tau ratio may therefore potentially be representative of a heterogeneous group of diagnostically unresolved patients, even though cutoff levels established within a population sample tend to overestimate their true diagnostic accuracy [29].

However, Buchhave et al. [32] reported a somewhat lower optimized cutoff value with regard to the $\operatorname{CSF} A \beta_{42} / p$-tau ratio of $<6.16$ (with a sensitivity of 0.88 and a specificity of 0.90 ) for predicting development of $\mathrm{AD}$ within 9.2 years in MCI patients. The observed difference 
Schjønning Nielsen et al.: Are CSF Biomarkers Useful as Prognostic Indicators in

Diagnostically Unresolved Cognitively Impaired Patients in a Normal Clinical Setting?

Table 3. Predictive ability of CSF biomarker cutoff values for future progression

\begin{tabular}{lllccc}
\hline & CSF A $\beta_{42}, \mathrm{pg} / \mathrm{ml}$ & $\mathrm{CSF}$-tau, $\mathrm{pg} / \mathrm{ml}$ & $\mathrm{CSF}$-tau, pg/ml & $\mathrm{A} \beta_{42} / \mathrm{p}$-tau & $\mathrm{t}$-tau $\times \mathrm{p}$-tau/A $\beta_{42}$ \\
\hline Cutoff value $^{\mathrm{a}}$ & 550 & $300 / 400$ & 80 & - & - \\
Sensitivity & 0.83 & 0.45 & 0.29 & - & - \\
Specificity & 0.60 & 0.88 & 0.93 & - & - \\
\hline Optimized cutoff value $^{\mathrm{b}}$ & 584 & 68 & 9.0 & 44.0 \\
Sensitivity $_{\text {Specificity }}$ & $0.86(0.81-0.90)$ & $0.53(0.47-0.59)$ & $0.41(0.35-0.47)$ & $0.72(0.67-0.78)$ & $0.64(0.58-0.70)$ \\
\hline
\end{tabular}

Sensitivity and specificity are presented with $95 \%$ confidence intervals. $\mathrm{CSF}=$ Cerebrospinal fluid; $\mathrm{A} \beta_{42}=\beta$-amyloid ar t-tau $=$ total tau; $p$-tau $=$ phosphorylated tau. ${ }^{a}$ Previously established cutoff value, sensitivity and specificity. ${ }^{\mathrm{b}}$ Optimized cutoff values are derived from ROC curve analysis by the cutoff that maximizes the sum of sensitivity and specificity.

may partly be due to the shorter follow-up period in our study, since some of the clinically stable patients might still progress during a longer follow-up period. Nevertheless, cutoff levels also tend to differ between studies [33]. This discrepancy is probably partly due to methodological variability and the absence of validated technical standard processes for CSF analysis [43].

Adding the t-tau protein as a biomarker of nonspecific neural damage did not add any discriminatory power over the $A \beta_{42} / p$-tau ratio for separating clinically progressive from clinically stable patients (fig. 3).

CSF neuroinflammation biomarkers are traditionally used in diagnostic workup primarily to exclude nonneurodegenerative disorders as causes of dementia, especially inflammatory diseases. In our population, only 1 patient was subsequently diagnosed with primary progressive multiple sclerosis.

Growing evidence indicates that unspecific neuroinflammation contributes to the pathophysiology of several neurodegenerative diseases including AD and related dementias [17, $20,44]$. Neuropathological findings suggest that cerebral inflammation plays a key role in the toxicity of amyloid and thus is part of an essential mechanism that determines which patients with intracerebral tau and amyloid pathology will become demented or stay nondemented. Other studies suggested that amyloid plaques and neurofibrillary tangles have the ability to initiate and sustain a chronic intracerebral inflammatory response $[19,20]$.

In our study, we did not find any indications for neuroinflammation in any of the groups. The fact that no increased signs of neuroinflammation or BBB dysfunction were found in clinically progressive diagnostically unresolved patients - and even in AD patients - compared to clinically stable patients was unexpected in light of the mounting evidence of the role of neuroinflammation in neurodegenerative dementias. This is quite in contrast to a recent study by Nägga et al. [20], which reported neuroinflammation to be independently linked to both rapidly progressive disease and early death in patients with AD. However, it is important to note that these authors found neuroinflammation to be independently linked to rapidly progressive disease and early death in patients with $\mathrm{AD}$, whereas the less aggressive courses of the disease may not exhibit same level of neuroinflammation.

Furthermore, Nägga et al. [20] found an elevated CSF/serum albumin ratio to be a predictor of death in $\mathrm{AD}$ patients. The $\mathrm{CSF} /$ serum albumin ratio is an indicator of $\mathrm{BBB}$ integrity. It has limited differential diagnostic power; nevertheless, it is a reliable sign of an active process [21-24, 45, 46]. Previous studies have reported conflicting evidence regarding findings of $\mathrm{BBB}$ dysfunction in neurodegenerative dementias, often attributing the presence of altered BBB integrity to concurrent neurovascular diseases [21, 23, 24]. However, evidence of altered BBB integrity in both neurodegenerative and vascular dementias has been established - even if BBB dysfunction was still found to be more pronounced in vascular dementia than in $\mathrm{AD}[45,46]$. 
There were no compelling signs of BBB dysfunction (i.e. abnormal CSF/serum albumin ratio or IgG index) in any of the groups of our study. Likewise, the ROC curves revealed a poor performance of all CSF neuroinflammation parameters when used to predict future cognitive decline and clinical progression in our population of diagnostically unresolved patients.

\section{Strengths and Limitations}

The strengths of our study are, first of all, its clinical setting, since we used a consecutively recruited, heterogeneous memory clinic population. Second, all diagnoses were established by consensus among an experienced multidisciplinary team based upon all the available examination results, and the diagnoses were reevaluated on an ongoing basis. Third, all lumbar punctures were performed by experienced physicians at 2 memory clinics, with established standard procedures for the lumbar puncture and subsequent sample handling. Finally, the CSF AD biomarker analyses were all carried out at one central laboratory.

A potential limitation of our study is the risk of circularity. The lumbar punctures and CSF analyses were performed in a clinical setting during the diagnostic workup, and the results were used in the diagnostic consensus-building process. This may have influenced diagnoses both at baseline and during the ongoing clinical evaluation, but not with regard to whether patients clinically progressed during follow-up. The variability in follow-up periods in our study was high. This may particularly be an issue with regard to the clinically stable group, as it is well established that time to conversion varies within a wide range, and it is likely that some of the patients labelled clinically stable in our study will eventually convert to having a neurodegenerative disease $[29,33]$. The variability in follow-up periods is partly due to the fact that shortly after their initial diagnosis, a few patients were reevaluated and found without cognitive complaints or to suffer from nondegenerative diseases, and thus were lost to follow-up. Our study may consequently underestimate the predictive value of the biomarkers assessed.

\section{Conclusion}

We investigated a group of patients with cognitive impairment who, even after an extensive diagnostic assessment involving CSF biomarkers, remained diagnostically unresolved as to the underlying cause of their impairment. We found that baseline CSF AD biomarkers - but not CSF neuroinflammation parameters - had the potential to predict clinical progression, regardless of the underlying condition.

Furthermore, the CSF A $\beta_{42} / p$-tau ratio showed both high discriminative power in separating progressive from stable diagnostically unresolved patients and a great ability to predict $\mathrm{AD}$ as the underlying neurodegenerative disease. This implies that the CSF $A \beta_{42} / p$-tau ratio might potentially be superior to the CSF AD biomarkers individually in predicting continuous cognitive decline and clinical progression in a group of initially diagnostically unresolved patients.

Our findings also suggest that a large proportion of initially diagnostically unresolved patients might in fact present with early AD with atypical or subtle symptoms and signs. Our findings may be clinically relevant in improving prognostic measures for initially diagnostically unresolved patients, but they are also relevant in identifying suitable patients for clinical trials of disease-modifying agents, which should be administered very early in the clinical course. More longitudinal studies of the diagnostic and predictive performance of CSF biomarkers in diagnostically unresolved patients with longer follow-up periods are needed to support our findings. 


\section{Acknowledgments}

This work was supported by grants from the Velux Foundations.

\section{Disclosure Statement}

The authors declare no conflicts of interest.

\section{References}

1 Prince M, Bryce R, Albanese E, Wimo A, Ribeiro W, Ferri CP: The global prevalence of dementia: a systematic review and metaanalysis. Alzheimers Dement 2013;9:63-75.e2.

-2 Sperling RA, Jack CR Jr, Aisen PS: Testing the right target and right drug at the right stage. Sci Transl Med 2011; 3:111 cm33.

-3 Ganguli M, Dodge HH, Shen C, DeKosky ST: Mild cognitive impairment, amnestic type: an epidemiologic study. Neurology 2004;63:115-121.

-4 Palmer K, Wang HX, Bäckman L, Winblad B, Fratiglioni L: Differential evolution of cognitive impairment in nondemented older persons: results from the Kungsholmen Project. Am J Psychiatry 2002;159:436-442.

-5 Solfrizzi V, Panza F, Colacicco AM, D’Introno A, Capurso C, Torres F, et al: Vascular risk factors, incidence of $\mathrm{MCI}$, and rates of progression to dementia. Neurology 2004;63:1882-1891.

6 Crystal HA, Dickson D, Davies P, Masur D, Grober E, Lipton RB: The relative frequency of 'dementia of unknown etiology' increases with age and is nearly 50\% in nonagenarians. Arch Neurol 2000;57:713-719.

7 McKhann GM, Knopman DS, Chertkow H, Hymann BT, Jack CR Jr, Kawas CH, et al: The diagnosis of dementia due to Alzheimer's disease: recommendations from the National Institute on Aging-Alzheimer's Association workgroups on diagnostic guidelines for Alzheimer's disease. Alzheimers Dement 2011;7:263-269.

8 World Health Organization: The ICD-10 Classification of Mental and Behavioural Disorders: Clinical Descriptions and Diagnostic Guidelines. Geneva, World Health Organization, 1992.

-9 Dubois B, Feldman HH, Jacova C, DeKosky ST, Barberger-Gateau P, Cummings J, et al: Research criteria for the diagnosis of Alzheimer's disease: revising the NINCDS-ADRDA criteria. Lancet Neurol 2007;6:734-746.

10 Solfrizzi V, D'Introno A, Colacicco AM, Capurso C, Todarello 0, Pellicani V, et al: Circulating biomarkers of cognitive decline and dementia. Clin Chim Acta 2006;364:91-112.

11 Mormino EC, Kluth JT, Madison CM, Rabinovici GD, Baker SL, Miller BL, et al: Episodic memory loss is related to hippocampal-mediated $\beta$-amyloid deposition in elderly subjects. Brain 2009;132(pt 5):1310-1323.

12 Jansen WJ, Ossenkoppele R, Knol DL, Tijms BM, Scheltens P, Verhey FR, et al: Prevalence of cerebral amyloid pathology in persons without dementia: a meta-analysis. JAMA 2015;313:1924-1938.

13 Kester MI, van der Vlies AE, Blankenstein MA, Pijnenburg YAL, van Elk EJ, Scheltens P, et al: CSF biomarkers predict rate of cognitive decline in Alzheimer disease. Neurology 2009;73:1353-1358.

14 Price JL, McKeel DW, Buckles VD, Roe CM, Xiong C, Grundman M, et al: Neuropathology of nondemented aging: presumptive evidence for preclinical Alzheimer disease. Neurobiol Aging 2009;30:1026-1036.

15 Price JL, Morris JC: Tangles and plaques in nondemented aging and 'preclinical' Alzheimer's disease. Ann Neurol 1999; 45:358-368.

16 Albert MS, DeKosky ST, Dickson D, Dubois B, Feldman HH, Fox NC, et al: The diagnosis of mild cognitive impairment due to Alzheimer's disease: recommendations from the National Institute on Aging-Alzheimer's Association workgroups on diagnostic guidelines for Alzheimer's disease. Alzheimers Dement 2011;7:270279.

17 Leonard BE: Inflammation, depression and dementia: are they connected? Neurochem Res 2007;32:17491756.

18 Strohmeyer R, Rogers J: Molecular and cellular mediators of Alzheimer's disease inflammation. J Alzheimers Dis 2001;3:131-157.

19 Shen Y, Lue L-F, Yang L-B, Roher A, Kuo Y-M, Strohmeyer R, et al: Complement activation by neurofibrillary tangles in Alzheimer's disease. Neurosci Lett 2001;305:165-168.

-20 Nägga K, Wattmo C, Zhang Y, Wahlund LO, Palmqvist S: Cerebral inflammation is an underlying mechanism of early death in Alzheimer's disease: a 13-year cause-specific multivariate mortality study. Alzheimers Res Ther 2014;6:41.

-21 Jesse S, Brettschneider J, Süssmuth SD, Landwehrmeyer BG, von Arnim CA, Ludolph AC, et al: Summary of cerebrospinal fluid routine parameters in neurodegenerative diseases. J Neurol 2011;258:1034-1041.

-22 Reiber H, Peter JB: Cerebrospinal fluid analysis: disease-related data patterns and evaluation programs. J Neurol Sci 2001;184:101-122.

23 Frölich L, Kornhuber J, Ihl R, Fritze J, Maurer K, Riederer P: Integrity of the blood-CSF barrier in dementia of Alzheimer type: CSF/serum ratios of albumin and IgG. Eur Arch Psychiatry Clin Neurosci 1991;240:363-366. 
24 Erickson MA, Banks WA: Blood-brain barrier dysfunction as a cause and consequence of Alzheimer's disease. J Cereb Blood Flow Metab 2013;33:1500-1513.

25 Winblad B, Palmer K, Kivipelto M, Jelic V, Fratiglioni L, Wahlund LO, et al: Mild cognitive impairment - beyond controversies, towards a consensus: report of the International Working Group on Mild Cognitive Impairment. J Intern Med 2004;256:240-246.

-26 Sjögren M, Vanderstichele H, Agren H, Zachrisson O, Edsbagge M, Wikkelsø C, et al: Tau and A $\beta 42$ in cerebrospinal fluid from healthy adults 21-93 years of age: establishment of reference values. Clin Chem 2001;47: 1776-1781.

27 Zwan M, van Harten A, Ossenkoppele R, Bouwman F, Teunissen C, Adriaanse S, et al: Concordance between cerebrospinal fluid biomarkers and $\left[{ }^{11} \mathrm{C}\right] \mathrm{PIB}$ PET in a memory clinic cohort. J Alzheimers Dis 2014;41:801807.

28 Mulder C, Verwey NA, van der Flier WM, Bouwman FH, Kok A, van Elk EJ, et al: Amyloid- $\beta(1-42)$, total tau, and phosphorylated tau as cerebrospinal fluid biomarkers for the diagnosis of Alzheimer disease. Clin Chem 2010; 56:248-253.

-29 Hampel H, Teipel SJ, Fuchsberger T, Andreasen N, Wiltfang J, Otto M, et al: Value of CSF $\beta$-amyloid and $_{12}$ and tau as predictors of Alzheimer's disease in patients with mild cognitive impairment. Mol Psychiatry 2004;9:705710.

-30 Kester MI, Boelaarts L, Bouwman FH, Vogels RL, Groot ER, Van Elk EJ, et al: Diagnostic impact of CSF biomarkers in a local hospital memory clinic. Dement Geriatr Cogn Disord 2010;29:491-497.

-31 Höglund K, Fourier A, Perret-Liaudet A, Zetterberg H, Blennow K, Portelius E: Alzheimer's disease - recent biomarker developments in relation to updated diagnostic criteria. Clin Chim Acta 2015;449:3-8.

-32 Buchhave P, Minthon L, Zetterberg H, Wallin AK, Blennow K, Hansson O: Cerebrospinal fluid levels of $\beta$-amyloid 1-42, but not of tau, are fully changed already 5 to 10 years before the onset of Alzheimer dementia. Arch Gen Psychiatry 2012;69:98-106.

-33 Hansson O, Zetterberg H, Buchhave P, Londos E, Blennow K, Minthon L: Association between CSF biomarkers and incipient Alzheimer's disease in patients with mild cognitive impairment: a follow-up study. Lancet Neurol 2006;5:228-234.

-34 Skillbäck T, Farahmand BY, Rosén C, Mattsson N, Nägga K, Kilander L, et al: Cerebrospinal fluid tau and amyloid- $\beta_{1-42}$ in patients with dementia. Brain 2015;138(pt 9):2716-2731.

- 35 Ferreira D, Rivero-Santana A, Perestelo-Pérez L, Westman E, Wahlund LO, Sarría A, et al: Improving CSF biomarkers' performance for predicting progression from mild cognitive impairment to Alzheimer's disease by considering different confounding factors: a meta-analysis. Front Aging Neurosci 2014;6:287.

-36 Genius J, Klafki H, Benninghoff J, Esselmann H, Wiltfang J: Current application of neurochemical biomarkers in the prediction and differential diagnosis of Alzheimer's disease and other neurodegenerative dementias. Eur Arch Psychiatry Clin Neurosci 2012;262(suppl 2):S71-S77.

-37 Jack CR, Knopman DS, Jagust WJ, Petersen RC, Weiner MW, Aisen PS, et al: Tracking pathophysiological processes in Alzheimer's disease: an updated hypothetical model of dynamic biomarkers. Lancet Neurol 2013; 12:207-216.

-38 Degerman Gunnarsson M, Lannfelt L, Ingelsson M, Basun H, Kilander L: High tau levels in cerebrospinal fluid predict rapid decline and increased dementia mortality in Alzheimer's disease. Dement Geriatr Cogn Disord 2014;37:196-206.

-39 Vos S, van Rossum I, Burns L, Knol D, Scheltens P, Soininen H, et al: Test sequence of CSF and MRI biomarkers for prediction of AD in subjects with MCI. Neurobiol Aging 2012;33:2272-2281.

40 Mattsson N, Zetterberg H, Hansson 0, Andreasen N, Parnetti L, Jonsson M, et al: CSF biomarkers and incipient Alzheimer disease in patients with mild cognitive impairment. JAMA 2009;302:385-393.

-41 van Rossum IA, Vos S, Handels R, Visser PJ: Biomarkers as predictors for conversion from mild cognitive impairment to Alzheimer-type dementia: implications for trial design. J Alzheimers Dis 2010;20:881-891.

-42 Zwan MD, Rinne JO, Hasselbalch SG, Nordberg A, Lleó A, Herukka SK, et al: Use of amyloid-PET to determine cutpoints for CSF markers. Neurology 2016;86:50-58.

43 del Campo M, Mollenhauer B, Bertolotto A, Engelborghs S, Hampel H, Simonsen AH, et al: Recommendations to standardize preanalytical confounding factors in Alzheimer's and Parkinson's disease cerebrospinal fluid biomarkers: an update. Biomark Med 2012;6:419-430.

44 Fakhoury M: Role of immunity and inflammation in the pathophysiology of neurodegenerative diseases. Neurodegener Dis 2015;15:63-69.

45 Popescu BO, Toescu EC, Popescu LM, Bajenaru O, Muresanu DF, Schultzberg M, et al: Blood-brain barrier alterations in ageing and dementia. J Neurol Sci 2009;283:99-106.

46 Iadecola C: The overlap between neurodegenerative and vascular factors in the pathogenesis of dementia. Acta Neuropathol 2010;120:287-296. 